One researcher's impressions of Graphene 2012

\section{Mildred S. Dresselhaus}

$\mathbf{M}$ any conferences on graphene have been held this calendar year and I myself have attended quite a few of them. For me, Graphene 2012held in Brussels on April 10-13-was in some ways similar to many of these other conferences, and in other ways it was a distinctive conference. Just as in the other conferences, the uniqueness of this wonderful material graphene was emphasized in the vast majority of the oral invited talks. This is not surprising because most graphene researchers are attracted to this field just because of the exceptional and intriguing properties of this material. Graphene is one atom in thickness and yet is robust enough to be interrogated repeatedly by thousands of researchers eager to learn more about its properties. An even larger number of engineers are eager to develop new presently unimaginable products based on graphene.

But Graphene 2012 was in fact different for me. Unlike other conferences, Graphene 2012 focused on the possibility that this novel material had in serving as a platform for a new Flagship research program aimed at advancing European research and development (R\&D) to a new and much higher activity level. The concept of the Flagship initiative is to provide sufficient resources to one or two specially selected research areas to make Europe far more competitive than it is currently in $R \& D$, and in this way to make Europe an international leader in the commercialization of the products stemming from the technologies that will be based on graphene.

The resource level envisaged for the Flagship program is $€ 1$ billion starting in 2013, with a ramp-up period of 2.5 years reaching $€ 100$ million per year in 2016 for a 7.5-year period going beyond 2020. The flagship program will include universities, industry, and government working collaboratively within the European community. The details of the proposed plan were not presented fully and may not yet have been fully decided at the time of composing this article, but the broad scope of the program was presented by Jari Kinaret of the Chalmers Institute of Technology in Sweden.

About 100 research groups are expected to participate in the Flagship program under 11 international work programs, with each work program headed by a leader. With 100 research groups participating, it would be expected that each leader would be responsible for managing a program with nine participating research groups on average. Each of the 11 leaders would be heading up a focused R\&D activity that would integrate the participating researchers doing the basic research with industrial groups working with the academic groups on applications.

The manner of carrying out the R\&D would be more like my vision of what happened historically in developments like radar, lasers, and the integrated circuit. The Flagship program will have a new approach for doing large-scale collaborative research across international borders and for funding such research. They will develop new approaches for decision-making, and project management. A Flagship program of this type will have an impact on those working both within and outside the program, both within Europe and elsewhere, and this includes citizens who are non-scientists, engineers, workers, and students worldwide.
One type of organization that is expected to emerge is an international conference series called Graphene 20XX, starting with the Graphene Conference in Bilbao in 2011 called ImagineNano. This conference attracted 1500 participants internationally from academia, industry, and government, with 450 attendees interested in graphene. The attendance of 650 participants in Graphene 2012 shows the present growing interest in graphene.

One obvious source of future participants would be the carbon nanotube community, because of some commonality of the science base between graphene and carbon nanotubes. On this issue my opinions should not be seriously considered because carbon nanotubes come into my own background. Clearly, graphene is attractive to a much larger audience than those already knowledgeable about nanocarbons. It is also noted here that Graphene 2012 took place in Brussels, which is an administrative center of the European Union, thereby providing a special overall European symbolism to the Graphene 2012 meeting that had a majority of European attendees.

Next, I would like to comment on the scientific content of Graphene 2012, because the participants were explicitly asked to provide input on the Flagship program, which seemed to me to be on the minds of many of the attendees, whether or not they were involved with the preparation of the Flagship proposal. Therefore, the implication was that in the near future the Graphene 2012 conference might be expected to play some role in the planning of future Graphene 20XX programs and conferences.

In preparing my remarks for the Conference Summary, I was subjected to the following constraints. I had only a couple hours' notice that I would be asked to give the conference summary. Therefore, the resulting product was mostly spontaneous and highly personal. It can therefore be said that this summary is what one person took away from attending the Graphene 2012 Conference. What follows are my personal perspectives on the scientific topics that impressed me the most. 


\section{Modifications of graphene by doping, defects, edges, and holes}

The structure of graphene can be modified in a number of ways and the properties are modified correspondingly. Prior studies distinguished doping by acceptors from doping by donors through carrier injection. Acceptor doping tends to expand the graphene lattice as additional electrons enter the lattice, while contraction occurs as electrons leave the lattice. Several presentations at this conference distinguished between one donor relative to another and similarly for distinguishing between different acceptors. Some interesting dopants are boron, nitrogen, and sulfur. Effective doping approaches through the use of positive and negative gates and by electrochemical potentials were also discussed.

Likewise, it has been common in the past for researchers to identify the presence of defects without specifying the particular type of defect structurally, and without distinguishing the effect that different defects have on the properties of graphene. For example, point defects, impurities of different kinds as just mentioned, grain boundaries, stacking faults, Stone-Thrower-Wales 5775 or 585 cluster defects, edge defects, graphene growth nucleation center defects, all have different structures and impart different crystalline perturbations. Progress has in fact been made in distinguishing one defect from another and here aberration-corrected high-resolution transmission electron microscopy (ACHRTEM) is now becoming increasingly available to distinguish one type of defect from another, but further work is needed to identify the defect type and then to consistently link an observed structural defect to the specific property modification that is caused by that defect. A good deal of progress has been made recently with the characterization of edges by the ACHRTEM technique. Luckily Raman spectroscopy provides a characteristic distinction between armchair and zigzag edges, which could in principle be checked against the characteristic structural differences for armchair and zigzag edges on the same samples measured by
ACHRTEM. At Graphene 2012 , an interesting presentation was given on holes (absence of a cluster of carbon atoms) in a graphene sheet with preferences made for holes with geometrical zigzag and armchair boundaries.

\section{Different kinds of samples}

Few-layer graphene samples differ somewhat according to their growth method. Graphene 2012 had a significant number of talks emphasizing chemical vapor deposition growth, and the study of monolayer graphene samples based on the carbon face of $\mathrm{SiC}$. For me this trend was new at an international conference, especially concerning the large number of talks presented on samples produced by this growth method.

Some discussion at Graphene 2012 addressed the use of intercalation as a method for preparing different kinds of graphene samples. The intercalation of graphene is already a well-developed topic historically, but what was new in recent years was the intercalation of fewlayer graphene and the intercalation of graphene-like materials. Regarding the intercalation of few-layer graphene is the adsorption of layers of other specific species between the lowest graphene layer and the surface, and the adsorption of specific other layers above the top surface of the few-layer graphene. In such scenarios it is possible to insert species that have not until now been introduced in a stable way either between the layers as an intercalated species or between the substrate and the initial layer, for which some examples of such foreign species are hydrogen, oxygen, and water. Repeated periodic superlattices of such structures based on graphene were shown. Periodic superlattices involving different layered materials, such as BN, $\mathrm{MoS}_{2}, \mathrm{WS}_{2}$, and several other layered materials were also discussed.

Though little discussion focused on graphene flakes, this topic did appear in the Graphene 2012 conference from both a structural and functional point of view. The interaction between graphene and a substrate was discussed, and the use of graphite or few graphene layer substrates for graphene flakes was popular.
Some discussion of the method of flake preparation took place as well as the relation between the preparation method and the different flake properties. The observation of special flake-flake interactions and flake-substrate interactions was noted.

\section{Bottom-up approaches}

Here two different research directions were highlighted. The chemistry approach allowed synthetic chemists to expand upon nanocarbon structures based on basic hydrocarbon structures. Starting with a pentacene building block, chemists can build a large hierarchy of follow-on structure. A future to such an approach can now be elaborated by starting with many different hydrocarbon building blocks thereby leading to a variety of interesting networks. In this way we are not bounded by nanotubes or graphene, but we can envisage other related scenarios based on, for example, wiggle structures of various kinds or even three-dimensional analogues of two-dimensional structures. Chemists could also generate a large variety of bottom-up scenarios that could be implemented and extended through mixing and matching.

Throughout the Graphene 2012 conference many research groups referred to $\mathrm{MoS}_{2}, \mathrm{WS}_{2}, \mathrm{Bi}_{2} \mathrm{Te}_{3}, \mathrm{Bi}_{2} \mathrm{Se}_{3}$, and a variety of other such layered materials which could be elaborated in ways that are related to what has already been published for few-layer graphene. However, there was little detailed elaboration on these very interesting materials. These elaborations, however, may well become topics for the future, and may lead to more interesting applications. Although many speakers emphasized the importance of two carbon sites A and B in the graphene lattice, little discussion was given to making the two sites different from one another in a controlled way.

For more information about the conference, go to www.grapheneconf.com. 


\section{Univaled EDS for TEN}

$0.98 s \mathrm{sr} 100 \mathrm{~mm}^{2}$

- Exponentially enhances elemental mapping for nano-area analysis TEM

- Automatic retractable design
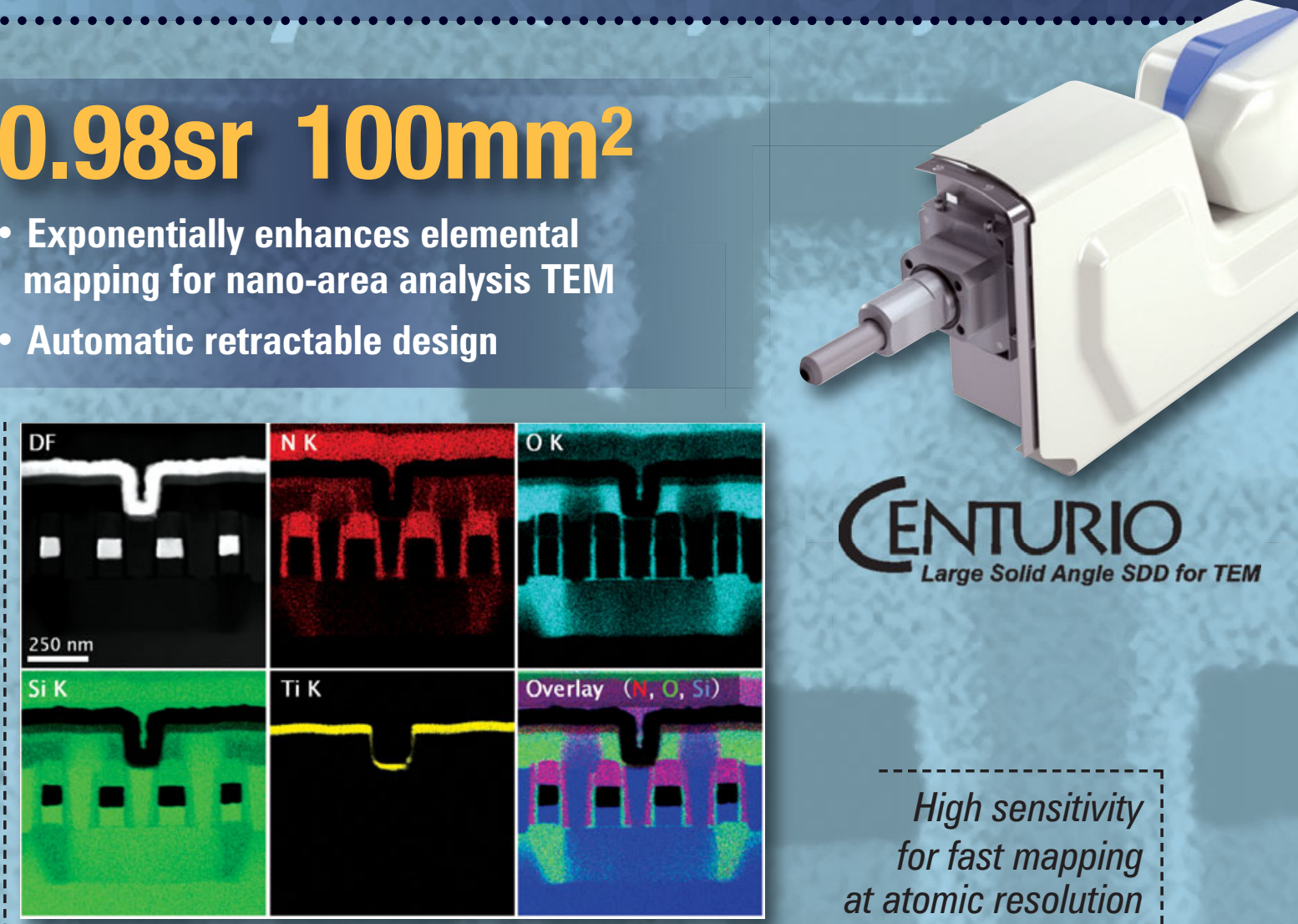

Seamless chemical mapping and data collection for S/TEM-TEM-

SEM-EDS. $(256 \times 256$ pixels.

Total acquisition time: $1 \mathrm{~min} .13 \mathrm{sec}$.

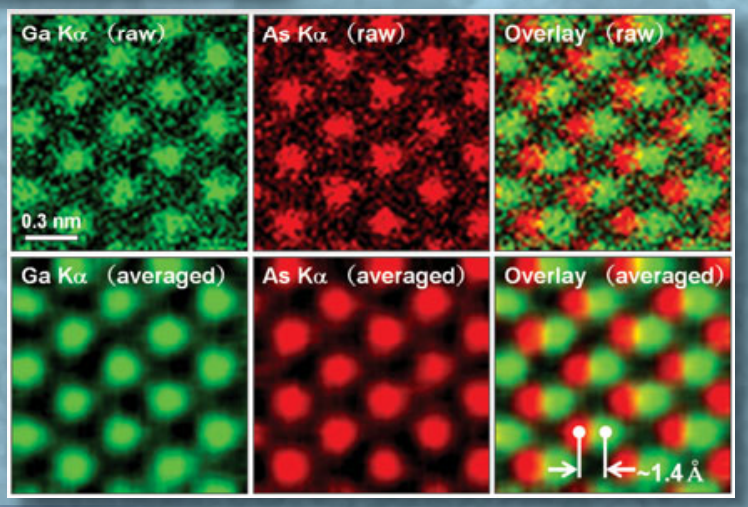

High sensitivity

for fast mapping at atomic resolution

View our real-time

DRAM analysis video

at WWw.jeolusa.com/UnrivaledEDS

\section{LEDL}

Global Solutions Provider for Advanced Technology

www.jeolusa.com•salesinfo@jeol.com 978-535-5900

Find us on Facebook and Twitter @jeolusa

JEM-2800
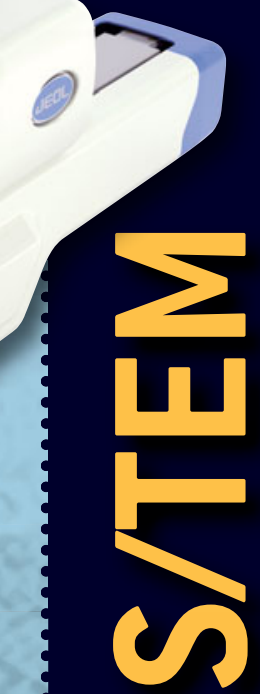

$\boldsymbol{C}$
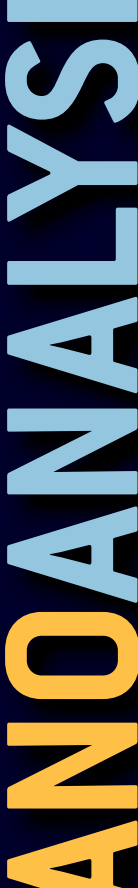